\title{
Relação das pessoas e instituições que doaram obras ou revistas á Biblioteca da Faculdade, durante o periodo compreendido entre 15 de Novembro de 1934 e 15 de Fevereiro de 1935
}

Aben Attar Nietto

Academia de Jurisprudencia da Catalunia

Addalzira Bittencourt

Affonso de Freitas Junior

Affonso de Taunay

Affonso de Toledo Bandeira de Mello

A. Gabriel da Veiga

Alcantara Machado

Alde Sampaio

Alice Toledo Tibiriçá

Amelia Duarte

A. Mesquita Carvalhaes

Anastacio Vasquez

Anibal Freire da Fonseca

Antonio Pires da Silva Machado

Antonio S. de Bustamante y Siven

A. Pompêo

Áriel, Editora Ltda.

Arquivos Brasileiros de Higiene Mental

Arthur de Vasconcelos

Associação dos Funcionarios Publicos do Estado de São Paủo Associação dos Proprietarios de São Paulo

Astolfo Vieira de Rezende
Atugasmin Medici Filho

Benito A. Nazar Anchorena

Bernardo Echieverri

Bertho Condé

Biblioteca del Collegio d'Avocats de Barcelona

Biblioteca Ida Faculdade de Medicina da Univiersidade de São Paulo

Biblioteca Nacional de Buenos Aires

Bibliotleca Nacional de Lisboa

Biblioteca Riograndense

Biblioteca de L'Ecole des Sciences Politiques de Paris

Caixa Economica Federal da Baía

Candido Oliveira Filho

Carlos C. Godoy

Carnegie Endowment for International Peace, (U.S.A.)

Celso Spinola

Cientre Européen de La Dotation Carnegie

Centro XI de Agosto

Cincinato Braga

Clovis Bevilaqua

Colegio de Abogados de Buenos Aires 
Comissión Protectora de Bibliotecas Populares de la Republica Argentina

Consul Geral da Alemanha em São Paulo

Consul Geral do Japão em São Paulo

Côrte de Apelação do Rio Grande do Sul

Departamento Nacional do Café

Diretoria de Estatistica Econom:ca e Financeira do Tesouro Nacional

Diretoria de Publicidade Agricola

Diretoria da Santa Casa de Misericordia de São Paulo

Editor Verlag Edanee

Editorial Reuss, S. A.

Embaixada do Mexico no Rio de Janeiro

E. M. de Carvalho Borges

Enéas Pinheiro

Enrique Gil

Eurico Teixeira Leite

Everardo Vallim Pereira de Souza

Fabio Pinto Coelho

Faculdade de Ciencias Juridicas e Sociais de La Plata

Faculdade de Direito da Baia

Faculdade de Direito e' Ciencias Sociais de Buenos Aires

Faculdade de Medicina de Porto Alegre

Flaminio Favero

Florencio Sanchez M. Rivas

Gabinete de Leitura Goiano

Gastão Cruls

G. B. Funaioli

Gennaro Escobedo

Georges Ottlik

Ginasio Pernambucano

Giorgio Del Vecchio

Gotarldo Pedemonte

Guia Fiscal
Heinrich Kalthoff

Heraclito Carneiro Ribeiro

IImprensa Oficial do Esstado de São Paulo

Instituto de Café do Estado de S. Paulo

Instituto Historico e Geografico da Baía

Instituto Historico e Geografico da do Espirito Santo

Instituto de Engenharia de São Paulo

Instituto de Fisica do Globo de Paris

Instituto de Identificação do Rio de Janeiro.

Instituto da Ordem dos Advogados do Rio Grande do Sul

Instituto de Organização Racional do Trabalho

Instituto Paulista de Contabilidade

Interventoria de Goiaz

Izabel Lobo Leite Ribeiro

James Ferraz Alvim

J. Caeiro da Matta

J. C. Ataliba Nogueira

J. F. Moreno

J. M. Vilhena Barbosa de Magalhães

João Edmundo Caldeira Brant

João da Gama Ceriqueira

João Henrique

João Rodrigues de Mereje

Jornal do Comercio

José Carlos de Macedo Soares

José Enrique Rodó

José Joaquim Cardoso de Mello Junior

José Joaquim Cardoso de Mello Netto

J. Pantoja Leite

Julio de Barros

Julio R. Agote

Julio Pevoredo 
Justo de Moraes

Laurenio Lago

La Revista Economica Sudamericana

Léo V:az

Leopoldo Teixeira Leite Filho

Les Editions du Cerf

Luiz Barbosa da Gama Cerqueira

Mackenzie College

Magarinos Torres

Manoel Martins Pacheco Prates

Mario Carrara

Ministerio da Agricultura

Mìnisterio das Corporações do Reino da Italia

Ministerio da Educação e Saude Publica

Ministerio da Fazenda

Ministerio da Justiça

M. Roiter

Museu Nacional do Rio de Janeiro

Museu Social de Paris

Nouvelle Revue de Hongrie

Octavio Morató Rodrigues

Odilon Braga

Oregon Law Review

Osvaldo Loudet

Partido Liberal Academico

Paulo Americo Pasalacqua

P. Leonardi Catholica

Political Science Quarterly

Poty Medeiros

Prefeitura Municipal de ISão Paulo

Raimundo Borch

Ranulpho Pinheiro Lima

Reitoria da Universidade de São Paulo

Revista Academica da Faculdade de Direito de Recife
Revista de Criminologia, Psiquiatria y Medicina Legal

Revista Javeriana

Revista de Jurisprudencia Brasi-leira

Revista Militar Brasileira

Revista Numismatica

Reynaldio Porchat

R. Instituto Superiore Navale dí Napoli

Roberto Simonsen

R. Scuola Superiore de Architettu-ra di Torino

Ruy de Azeveldo Sodré

Sadalla Amin Ghanem

Secção de São Paulo da Ordem dos Advogados Brasileiros

Secretaria da Faculdade de Dirleito da Universidade de São Paulo

Secretaria da Relação Exterior do Panamá

Secretaria da Sociedade de Geografia de Lisboa

Seminario de Ciencias Juridicas e Sociais

Sergio Milliet da Costa e Silva

Sociedade Anonima Edizione Remo Sandron de Palermo

Spencer Vampré

Sociedade das Nações

Sud Mennucci

The Harvard Law Review Association

Theodor Niemeyer

Tristão de Athayde

União Panamericana

Universidade de Azuay

Universidade de Buenos Aires

Universidade de Cahen

Universidade Central da Bolivia: 
Universidade de Chile

Universidade de Granada

Universidade de Havana

Universidade Imperial de Kyoto

Universidade de Kiel

Universidade de Mexico

Universidade Tecnica do

Grande do Sul
Universidade de Urbino

Universidade do Uruguay

Vanitas

Vicente de Azevedo

Victor de Carvalho

Vieira Cavalcanti

do Rio Waldemar Ferreira

W. B. Berardinelli 